\title{
FACTORIZATIONS OF LEBESGUE MEASURE VIA CONVOLUTIONS
}

\author{
NORMAN LEVENBERG, JR., GAVEN J. MARTIN, ALLEN L. SHIELDS AND \\ SMILKA ZDRAVKOVSKA
}

(Communicated by Irwin Kra)

\begin{abstract}
Given a continuous, increasing function $\phi:[0, \infty) \rightarrow[0, \infty)$ with $\phi(0)=0$, we define the Hausdorff $\phi$-measure of a bounded set $E$ in the unit interval $I=[0,1]$ as $H_{\phi}(E)=\lim _{\delta \rightarrow 0} H_{\phi}, \delta(E)$ where $H_{\phi},{ }_{\delta} E=\inf \sum_{i=1}^{\infty} \phi\left(t_{i}\right)$ and the infimum is taken over all countable covers of $E$ by intervals $U_{i}$ with $t_{i}=\left|U_{i}\right|=$ length of $U_{i}<\delta$. We show that given any such $\phi$, there exist closed, nowhere dense sets $E_{1}, E_{2} \subset I$ with $H_{\phi}\left(E_{1}\right)=H_{\phi}\left(E_{2}\right)=0$ and $E_{1}+E_{2} \equiv\left\{a+b: a \in E_{1}, b \in E_{2}\right\}=I$. The sets $E_{i}(i=1,2)$ are constructed as Cantor-type sets $E_{i}=\bigcap_{n=1}^{\infty} E_{i, n}$ where $E_{i, n}$ is a finite union of disjoint closed intervals. In addition, we give a simple geometric proof that the natural probability measures $\mu_{i}$ supported on $E_{i}$ which arise as weak limits of normalized Lebesgue measure on $E_{i, n}$ have the property that the convolution $\mu_{1} * \mu_{2}$ is Lebesgue measure on $I$.
\end{abstract}

Introduction. In 1949, H. G. Eggleston [E] showed that there exist compact subsets $E_{1}$ and $E_{2}$ of the unit interval $I=[0,1]$ which have Hausdorff dimension zero, but nonetheless have the property that the algebraic sum $E_{1}+E_{2}=\{x+y: x \in$ $E_{1}$ and $\left.y \in E_{2}\right\}$ is the entire unit interval $I$.

Following the ideas of Eggleston, we generalize his result by showing that for any given continuous increasing function $\Phi$ with $\Phi(0)=0$, there are compact subsets $E_{1}$ and $E_{2}$ of the unit interval $I$ such that $E_{1}+E_{2}=I$ and the Hausdorff $\Phi$-measure of both $E_{1}$ and $E_{2}$ is zero.

In addition we show that the natural probability measures $\mu_{1}$ and $\mu_{2}$, supported on $E_{1}$ and $E_{2}$, are measures whose convolution $\mu_{1} * \mu_{2}$ is Lebesgue measure $\lambda$ on I. Thus $\mu_{1} * \mu_{2}=\lambda$ can be thought of as a nontrivial factorization of Lebesgue measure. In the last section we will consider such factorizations of other measures absolutely continuous with respect to Lebesgue measure.

We wish to thank R. Lyons for pointing out a probabilistic approach to the proof of Theorem 3.1, which we outline. We would also like to acknowledge that W. Rudin communicated to the first named author an independent proof of Theorem 3.1 and some of its consequences. It was also pointed out to us that a similar question was studied by Tortrat who obtained a version of Theorem 3.1; see [T].

Finally, we wish to thank the referee who pointed out an error in an earlier draft and provided us with some additional references.

1. We begin with the general construction of the sets $E_{1}$ and $E_{2}$. Given a sequence of positive integers greater than one $\left\{q_{i}\right\}_{i=1,2, \ldots}$ (by convention we set

Received by the editors Septermber 4, 1987. The contents of this paper were first presented in preliminary forms in January, 1986 (AMS \#825, New Orleans).

1980 Mathematics Subject Classification (1985 Revision). Primary 28A50, 28A35, 28 A33. 
$\left.q_{0}=1\right)$ we can express any number $t$ in the unit interval $I=[0,1]$ in the form

$$
t=\sum_{i=1}^{\infty} p_{i} /\left(q_{1} \cdot q_{2} \cdots q_{i}\right)
$$

where $\left\{p_{i}\right\}_{i=1,2, \ldots}$ is a sequence of integers satisfying $0 \leq p_{i}<q_{i}$.

One can see this fact geometrically by subdividing the interval into $q_{1}$ equal subintervals and each of those subintervals into $q_{2}$ further subintervals and so on. The number $p_{1}$ tells which of the first $q_{1}$ intervals the point lies, $p_{2}$ tells which further subinterval the point lies and so on (there is only an ambiguity at the endpoints of each subinterval and this is easily dealt with).

Let

$$
E_{1}=\left\{x \in I: x=\sum_{i=1}^{\infty} p_{2 i-1} /\left(q_{1} \cdot q_{2} \cdots q_{2 i-1}\right)\right\}
$$

and

$$
E_{2}=\left\{x \in I: x=\sum_{i=1}^{\infty} p_{2 i} /\left(q_{1} \cdot q_{2} \cdots q_{2 i}\right)\right\} .
$$

We make the following observations about the sets $E_{1}$ and $E_{2}$.

(A) $E_{1}+E_{2}=I$.

(B) The sets $E_{1}$ and $E_{2}$ are perfect, nowhere dense sets that may be written as

$$
E_{1}=\bigcap_{n=1}^{\infty} E_{1, n}, \quad E_{2}=\bigcap_{n=1}^{\infty} E_{2, n},
$$

where $E_{1,1} \supseteq E_{1,2} \supseteq E_{1,3} \supseteq \cdots$ and $E_{2,1} \supseteq E_{2,2} \supseteq E_{2,3} \supseteq \cdots$ are a nested sequence of closed sets and $E_{1, n}$ consists of $c_{n}=q_{1} \cdot q_{3} \cdots q_{2 n-1}$ disjoint intervals of length $1 /\left(q_{1} \cdot q_{2} \cdots q_{2 n}\right)$, and $E_{2, n}$ consists of $d_{n}=q_{2} \cdot q_{4} \cdots q_{2 n}$ disjoint intervals of length $1 /\left(q_{1} \cdot q_{2} \cdots q_{2 n+1}\right)$.

It is not difficult to see that $E_{i}, i=1,2$, is perfect (we may perturb the expansion to find a sequence converging to any given point). To simplify notation we set $r_{n}=q_{1} \cdot q_{2} \cdots q_{n}$. To see (B), we inductively construct the sets $E_{i, n}$. We construct $E_{1,1}$ by dividing $I$ into $q_{1}$ equal intervals of length $1 / q_{1} ; E_{1,1}$ is then

$$
E_{1,1}=\bigcup\left\{\left[j / q_{1}, j / q_{1}+1 / q_{1} \cdot q_{2}\right]: j=0,1,2, \ldots, q_{1}-1\right\} .
$$

Given $E_{1, n}$, we construct $E_{1, n+1}$ by subdividing each subinterval $I_{n}$ of $E_{1, n}$ (there are $c_{n}$ such intervals of length $1 / r_{2 n}$ ) into $q_{2 n+1}$ intervals of equal length and from each such interval we choose the leftmost subinterval $I_{n+1}$ of length $1 / r_{2 n+2}$. The union of all the intervals so chosen is then $E_{1, n+1}$ (see Figure 1).

We construct $E_{2, n}$ in a similar fashion. We first subdivide the interval $\left[0,1 / q_{1}\right]$ into $q_{2}$ subintervals of equal length $1 /\left(q_{1} \cdot q_{2}\right) ; E_{2,1}$ is then the union of $q_{2}$ closed subintervals

$$
E_{2,1}=\bigcup\left\{\left[j / r_{2}, j / r_{2}+1 / r_{3}\right]: j=0,1,2, \ldots, q_{2}-1\right\} .
$$

Then given $E_{2, n}$ we construct $E_{2, n+1}$ by subdividing each subinterval of $E_{2, n}$ (there are $d_{n}$ such subintervals each of length $1 / r_{2 n+1}$ ) into $q_{2 n+1}$ intervals of 


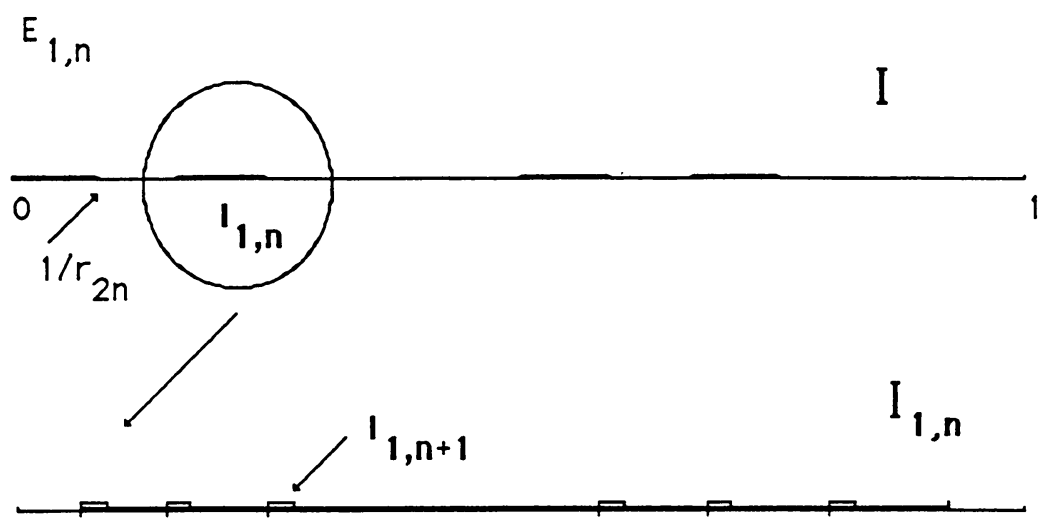

FIGURE 1

equal length and then choosing the leftmost subinterval of each such interval with length $1 / r_{2 n+3}$. The union of all the intervals so chosen is $E_{2, n+1}$.

It is now clear that $\left\{E_{i, n}\right\}_{n=1,2, \ldots}$ is a closed and nested sequence of sets. We set $F_{i}=\bigcap E_{i, n}$. We wish to show that $F_{i}$ is a closed, perfect, nowhere dense set and that $F_{i}=E_{i}$.

It is clear that $F_{i}$ is closed. Since the diameter of each component of $E_{i, n}$ has length no more than $1 / r_{2 n}$, we see that the diameter of each component of $F_{i}$ is zero. That is, $F_{i}$ is totally disconnected; and since it is closed, it is nowhere dense. Thus if we show $E_{i}=F_{i}$ we will have proved (B). (A) follows from the choice of $E_{1}$ and $E_{2}$.

We show that $E_{1}=F_{1} ; E_{2}=F_{2}$ will follow in a similar fashion. The set of left endpoints $L_{n}$ of the intervals of $E_{1, n}$ is

$$
L_{n}=\left\{x=\sum_{i=1}^{n} p_{2 i-1} / r_{2 i-1}: 0 \leq p_{2 i-1}<q_{2 i-1}\right\} \text {. }
$$

Thus $L_{n}$ lies in $E_{1}$ for all $n$. Hence $\bigcup L_{n}$ is contained in $E_{1}$. Since $\bigcup L_{n}$ is dense in $F_{1}$ and since $E_{1}$ is closed, we find $F_{1}$ is contained in $E_{1}$. Similarly, since the set of left endpoints is retained at each stage, $F_{1} \supseteq \cdots \supseteq L_{n+1} \supseteq L_{n}$, and since each point of $E_{1}$ is a limit of points of $\bigcup L_{n}$, we find $E_{1}$ is contained in $F_{1}$, since $F_{1}$ is closed. Hence $E_{1}=F_{1}$.

We now define probability measures $\mu_{i, n}$ supported on $E_{i, n}, i=1,2$, by using a constant multiple of Lebesgue measure on each subinterval $I_{i, n}$ of $E_{i, n}$. More precisely, we let $\chi_{i, n}$ be the characteristic function of $E_{i, n}$ and set

$$
\begin{gathered}
d \mu_{1, n}=q_{2} \cdot q_{4} \cdots q_{2 n} \cdot \chi_{1, n}(x) d \lambda(x), \\
d \mu_{2, n}=q_{1} \cdot q_{3} \cdots q_{2 n+1} \cdot \chi_{2, n}(x) d \lambda(x) .
\end{gathered}
$$

From our construction of the sets $E_{i, n}$ we find that the measures $\mu_{i, n}$ have the following properties:

$$
\begin{gathered}
\mu_{i, n}([0,1])=1, \quad n=1,2, \ldots \\
\mu_{i, n}\left(I_{i, n}\right)=\mu_{i, m}\left(I_{i, n}\right) \quad \text { for } m \geq n
\end{gathered}
$$




$$
\begin{aligned}
& \text { the measures }\left\{\mu_{i, n}\right\}_{n=1,2, \ldots} \text { converge weak* to a probability mea- } \\
& \text { sure } \mu_{i} \text { supported on } E_{i} \text {. }
\end{aligned}
$$

(D) can be rephrased by saying that the intervals $I_{i, n}$ carry the same mass from some finite stage onwards. This fact will ensure the weak* convergence of the measures indicated in $(\mathrm{E})$.

Henceforth, $E_{i}$ and $\mu_{i}$ will refer to the sets and measures obtained above from a given sequence $\left\{q_{n}\right\}$. We will show that an appropriate choice of the sequence $\left\{q_{n}\right\}$ we lead to some interesting consequences. See also [KS] for other related Cantor set constructions.

2. Let $\Phi:[0, \infty) \rightarrow[0, \infty)$ be any continuous increasing function with $\Phi(0)=0$. Given a bounded subset $E$ of the interval $I$, we define

$$
H_{\Phi, \delta}(E)=\inf \sum_{i=1}^{\infty} \Phi\left(t_{i}\right),
$$

where the infimum is taken over all countable covers of $E$ by intervals $U_{i}$ each of length $t_{i}=\lambda\left(U_{i}\right)<\delta$. Clearly $H_{\Phi, \delta}(E) \geq H_{\Phi, \delta^{\prime}}(E)$ if $\delta \leq \delta^{\prime}$, and hence we may define

$$
H_{\Phi}(E)=\lim _{\delta \rightarrow 0} H_{\Phi, \delta}(E)
$$

and call $H_{\Phi}(E)$ the $\Phi$-measure of $E$. For $\Phi(t)=t^{\alpha}, 0<\alpha \leq 1$, we obtain the classical $\alpha$-Hausdorff measure, $H_{\alpha}(E)$. If $H_{\alpha}(E)=0$ for $\alpha>\beta$ and $H_{\alpha}(E)=+\infty$ for $\alpha<\beta$, we say $E$ has Hausdorff dimension $\beta$. We now strengthen Eggleston's result with the following theorem.

2.1 THEOREM. Given any continuous increasing function $\Phi$ with $\Phi(0)=0$, we can construct subsets $E_{1}$ and $E_{2}$ of the unit interval such that

$$
H_{\Phi}\left(E_{1}\right)=H_{\Phi}\left(E_{2}\right)=0
$$

and

$$
E_{1}+E_{2}=I
$$

PrOOF. We construct a sequence $\left\{q_{k}\right\}$ inductively as follows. Set $q_{0}=1$. Given $q_{0}, q_{1}, \ldots, q_{n}$ we choose $q_{n+1}$ so large that

$$
\Phi\left(1 / q_{n+1}\right)<1 /\left(n \cdot r_{n}\right) .
$$

To show that $H_{\Phi}\left(E_{1}\right)=0$, it suffices to show that there is a sequence of positive numbers $\left\{\delta_{n}\right\}$, such that $\delta_{n} \rightarrow 0$ and that

$$
H_{\Phi, \delta_{n}}\left(E_{1}\right)=O(1 / n) \text {. }
$$

Since $E_{1, n}$ consists of $q_{1} \cdot q_{3} \cdots q_{2 n-1}$ intervals $I_{1, n}$ of length $1 /\left(q_{1} \cdot q_{2} \cdots q_{2 n}\right)=\delta_{n}$, and since $E_{1}$ is contained in $E_{1, n}$, we see that

$$
\begin{aligned}
H_{\Phi, \delta_{n}}\left(E_{1}\right) & \leq H_{\Phi, \delta_{n}}\left(E_{1, n}\right) \leq q_{1} \cdot q_{3} \cdots q_{2 n-1} \Phi\left(1 /\left(q_{1} \cdot q_{2} \cdots q_{2 n}\right)\right) \\
& \leq q_{1} \cdot q_{3} \cdots q_{2 n-1} \Phi\left(1 / q_{2 n}\right) \quad(\text { as } \Phi \text { is increasing }) \\
& \left.\leq q_{1} \cdot q_{3} \cdots q_{2 n-1} 1 /\left((2 n-1) q_{1} \cdot q_{2} \cdots q_{2 n-1}\right)\right) \\
& \leq 1 /(2 n-1) .
\end{aligned}
$$

A similar calculation shows that $H_{\Phi}\left(E_{2}\right)=0$. The fact that $E_{1}+E_{2}=I$ has been noted above. The proof is complete.

For a subset $E$ of the unit interval we define the logarithmic measure of $E$ as $L(E)=H_{\Phi}(E)$, where $\Phi(t)=1 / \log (1 / t)$. 
2.6 COROLLARY. There exist compact subsets $E_{1}$ and $E_{2}$ of the unit interval satisfying

$$
L\left(E_{1}\right)=L\left(E_{2}\right)=0
$$

and

$$
E_{1}+E_{2}=I .
$$

In particular, $L\left(E_{1}+E_{2}\right)=\infty$.

It is known (see [N, Chapter $5, \S 6$, p. 147]) that a set of logarithmic measure zero has logarithmic capacity zero. Thus Corollary 2.6 implies the existence of two compact sets $E_{1}$ and $E_{2}$ each with capacity zero and yet their algebraic sum, which is the whole interval, has positive capacity.

2.7 PROPOSITION. The measure $\mu_{1}$ associated with the set $E_{1}$ in Corollary 2.6 is an Evans measure for the set $E_{1}$. That is, the potential function

$$
P \mu_{1}(z)=\int_{E_{1}} \log (1 /|z-y|) d \mu_{1}(y)
$$

satisfies

$$
\begin{array}{ll}
P \mu_{1}(z)=+\infty & \text { for } z \in E_{1}, \\
P \mu_{1}(z)<+\infty & \text { for } z \in \mathbf{R}^{2}-E_{1} .
\end{array}
$$

Similarly, the measure $\mu_{2}$ is an Evans measure for the set $E_{2}$.

PROOF. Since $\mu_{1}$ is a probability measure supported on the compact set $E_{1}$, (2.9) is immediate. To prove (2.8) we fix an $x \in E_{1}$. In our construction of the sets $E_{1}$ and $E_{2}$ in Corollary 2.6 we chose integers $q_{1}, q_{2}, \ldots$ satisfying (2.4) for $\Phi(t)=1 / \log (1 / t)$. Thus

$$
\log \left(q_{n+1}\right)>n \cdot q_{1} \cdot q_{2} \cdots q_{n}
$$

Then for each $n$, there is a subinterval $I_{1, n}$ of $E_{1, n}$ which contains $x$. Now $0 \leq$ $x, y \leq 1$ implies that $\log (1 /|x-y|) \geq 0$ and so

$$
\begin{aligned}
P \mu_{1}(x) & \geq \int_{I_{1, n}} \log (1 /|x-y|) d \mu_{1}(y) \\
& \geq \log \left(1 / \lambda\left(I_{1, n}\right)\right) \mu_{1}\left(I_{1, n}\right) \\
& =\log \left(1 / \lambda\left(I_{1, n}\right)\right) \mu_{1, n}\left(I_{1, n}\right) \quad \text { by }(\mathrm{C}) \text { and }(\mathrm{D}) \\
& =\left(\log \left(q_{1} \cdot q_{2} \cdots q_{2 n}\right)\right) /\left(q_{1} \cdot q_{3} \cdots q_{2 n-1}\right) \\
& \geq \log \left(q_{2 n}\right) /\left(q_{1} \cdot q_{3} \cdots q_{2 n-1}\right) \\
& \geq 2 n-1 \quad \text { by }(2.10) .
\end{aligned}
$$

The result now follows.

3. We have seen that by choosing our sequence $\left\{q_{n}\right\}$ so that the $q_{n}$ 's increase sufficiently rapidly, we can construct very small sets $E_{1}$ and $E_{2}$ whose algebraic sum is the whole interval $I$. The convolution $\mu_{1} * \mu_{2}$ of the measures $\mu_{1}$ and $\mu_{2}$ is supported in the algebraic sum of the supports of $\mu_{1}$ and $\mu_{2}$, and thus in the whole interval. We now show that no matter how small the sets $E_{i}$ are, the measure $\mu_{1} * \mu_{2}$ is large. 
3.1 THEOREM. For any sequence $\left\{q_{n}\right\}_{n=1,2, \ldots}$ with $1<q_{1} \leq q_{2} \leq \cdots$ we have

$$
\mu_{1} * \mu_{2}=\lambda(=\text { Lebesgue measure on } I) \text {, }
$$

$$
\mu_{1, n} * \mu_{2, m}=\lambda \quad(\bmod 1) \quad \text { for any pair of positive integers } m \text { and } n \text {. }
$$

PROOF. Here mod 1 refers to the fact that the support of $\mu_{1, n} * \mu_{2, m}$ will extend slightly beyond the interval $I$; if we add this extra mass starting at the origin, we do indeed obtain Lebesgue measure. This problem could be circumvented by working on the unit circle, but this would then complicate the geometrically simple proof.

That (3.2) follows from (3.3) can be seen from the fact that $\mu_{i, n} \rightarrow \mu_{i}$ weak $^{*}$ and thus $\left(\mu_{1, n} \times \mu_{2, n}\right) \rightarrow\left(\mu_{1} \times \mu_{2}\right)$ weak $^{*}$ on the product space $E_{1} \times E_{2}$, together with the fact that the convolution is obtained by integrating on the product space. That is,

$$
\int f d\left(\mu_{1} * \mu_{2}\right)=\iint f(x+y) d \mu_{1}(x) d \mu_{2}(y) .
$$

We will prove that $\mu_{1, n} * \mu_{2, n}=\lambda$. The general case $\mu_{1, n} * \mu_{2, m}=\lambda$ will then follow from a simple geometric observation. We will outline the proof in the case that $n=m=1$. This case is typical of the general case and its outline will simplify and motivate the general discussion following.

The measure $\mu_{1,1}$ is supported on the $q_{1}$ intervals $I_{1,1}$ comprising $E_{1,1}$ and the measure $\mu_{2,1}$ is supported on the $q_{2}$ intervals $I_{2,1}$ comprising $E_{2,1}$. Hence the product measure $\mu_{1,1} \times \mu_{2,1}$ is supported on $q_{1} \cdot q_{2}$ rectangles which we denote by $R_{i, j}$. Also

$$
\left(\mu_{1,1} \times \mu_{2,1}\right)\left(R_{i, j}\right)=\mu_{1,1}\left(I_{1,1}\right) \cdot \mu_{2,1}\left(I_{2,1}\right)=1 /\left(q_{1} \cdot q_{2}\right) .
$$

We illustrate this in Figure 2 by putting $E_{1,1}$ on the vertical axis and $E_{2,1}$ on the horizontal axis.

We define $p(x, y)=x+y$. The diagonal lines in Figure 2 are to indicate the effect of projection via $p$. We compute the $\mu_{1,1} * \mu_{2,1}$ measure of a Borel subset $E$ of $I$ as

$$
\left.\left(\mu_{1,1} * \mu_{2,1}\right)(E)=\left(\mu_{1,1} \times \mu_{2,1}\right)\left(p^{-^{*}}{ }^{\prime} E\right)\right) \text {. }
$$

The idea of the proof that $\mu_{1,1} * \mu_{2,1}=\lambda$ is that the rectangles project down to exactly fill out the interval $I$. We compute the $\mu_{1,1} * \mu_{2,1}$ measure of $E$ as the sum

$$
\mu_{1,1} * \mu_{2,1}(E)=\sum_{i, j} q_{1} \cdot q_{2} \cdot q_{3} \cdot(\lambda \times \lambda)\left(p^{-1}(E) \cap R_{i, j}\right) .
$$

Now projecting the rectangles down the diagonal so that their bases lie on the horizontal axis will not change the two-dimensional Lebesgue measure of $p^{-1}(E) \cap$ $R_{i, j}$. After such projection, the rectangles will fill out the strip $Q_{1}=[0,1] \times$ $\left[0,1 /\left(q_{1} \cdot q_{2} \cdot q_{3}\right)\right]$. Then we find that the measure

$$
\begin{aligned}
\mu_{1,1} * \mu_{2,1}(E) & =q_{1} \cdot q_{2} \cdot q_{3} \cdot(\lambda \times \lambda)\left(p^{-1}(E) \cap Q_{1}\right) \\
& =r_{3} \cdot \lambda(E) \cdot\left(1 / r_{3}\right) \quad(\bmod 1) \\
& =\lambda(E) \quad(\bmod 1) .
\end{aligned}
$$

We need some notation and definitions to formalize the above discussion. For a rectangle $R=[a, b] \times[c, d]$ in $[0,1] \times[0,1]$ we define the projection along the diagonal $P(R)$ to be the rectangle

$$
P(R)=[a+c, b+c] \times[0, d-c] \quad(\bmod 1),
$$




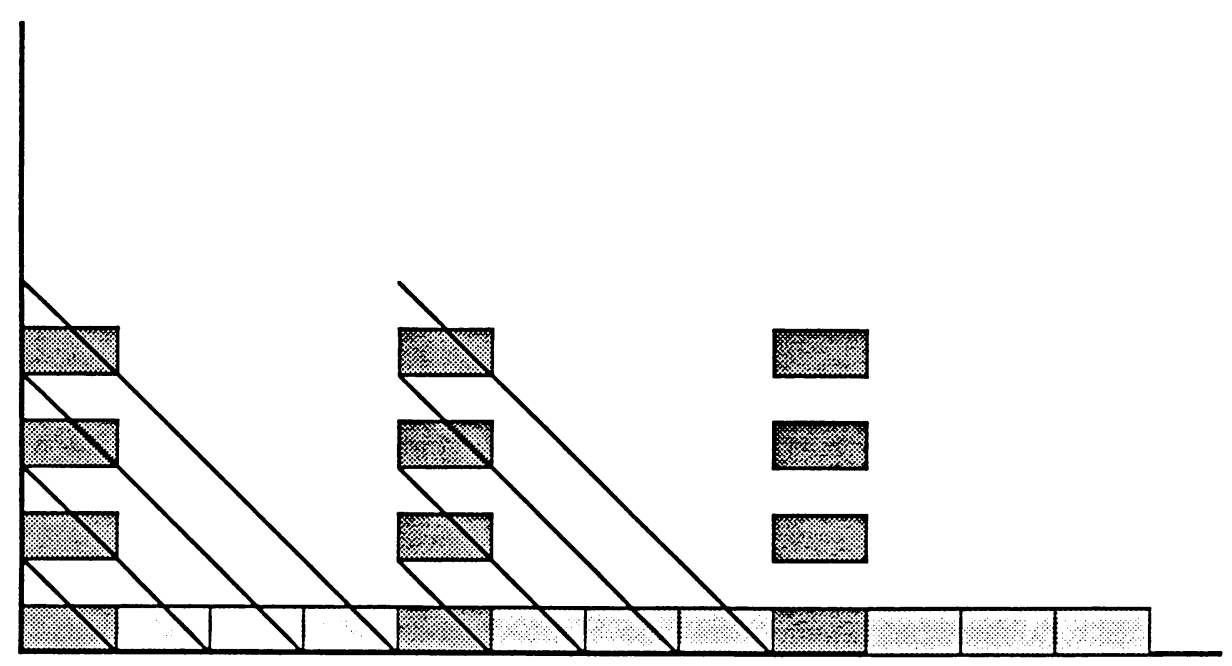

FIGURE 2

where again the mod 1 refers to the addition of the overlap to the origin.

We will consider the case $\mu_{1, n} * \mu_{2, n}$. Let $R$ be a rectangle in the support of $\mu_{1, n} \times \mu_{2, n}$. Then $R=I_{1, n} \times I_{2, n}$ for appropriate subintervals $I_{1, n}$ of $E_{1, n}$ and $I_{2, n}$ of $E_{2, n} . R$ is isometric to the rectangle

$$
\left[0,1 /\left(q_{1} \cdot q_{2} \cdots q_{2 n}\right)\right] \times\left[0,1 /\left(q_{1} \cdot q_{2} \cdots q_{2 n} \cdot q_{2 n+1}\right)\right]=\left[0,1 / r_{2 n}\right] \times\left[0,1 / r_{2 n+1}\right]
$$

and has total $\left(\mu_{1, n} \times \mu_{2, n}\right)$-mass $1 / r_{2 n}$. We set $Q_{n}=[0,1] \times\left[0,1 / r_{2 n+1}\right]$. We wish to show that the projection of all the rectangles precisely fills out $Q_{n}$ with no overlap of interior points. This will then prove (3.3) for the case $m=n$. We index all the rectangles in the support of $\mu_{1, n} \times \mu_{2, n}$ as $\left\{R^{n_{k}}\right\}_{k=1,2, \ldots}$.

3.5 Proposition. For each $n=0,1,2, \ldots$,

$$
\bigcup\left\{P\left(R^{n_{k}}\right): k=1,2, \ldots, r_{2 n}\right\}=Q_{n} .
$$

Futhermore,

$$
\operatorname{int}\left(P\left(R^{n_{k}}\right)\right) \cup \operatorname{int}\left(P\left(R^{n_{j}}\right)\right)=\varnothing, \quad k \neq j .
$$

Proof. We use induction. Since $q_{0}=1$ and $R^{0_{0}}=I \times\left[0, q_{1}\right]=P\left(R^{0_{0}}\right)=Q_{0}$, the case $n=0$ is immediate. We assume that (3.6) and (3.7) are both true for the index $n$. To prove (3.6) and (3.7) for the index $n+1$, it suffices to show that for each rectangle $R^{n_{k}}$, the projection of the subrectangles obtained from the $(n+1)$ st subdivision which lie in the rectangle $R^{n_{k}}$ cover the base of the projection of $R^{n_{k}}$ without overlap. More precisely, we wish to show that

$$
\begin{aligned}
& \text { if } P\left(R^{n_{k}}\right)=\left[a, a+1 / r_{2 n}\right] \times\left[0,1 / r_{2 n+1}\right] \\
& \text { then } \bigcup\left\{P\left(R^{n+1_{j}}\right): R^{n_{k}} \supseteq R^{n+1_{j}}\right\}=\left[a, a+1 / r_{2 n}\right] \times\left[0,1 / r_{2 n+3}\right] .
\end{aligned}
$$

One can see that this really is identical to the case occuring in the first subdivision, except that it is on a smaller rectangle. It is clear that we may assume for simplicity that $a=0$. Thus we set $W$ as the rectangle $W=\left[0,1 / r_{2 n}\right] \times\left[0,1 / r_{2 n+1}\right]$. 
One can think of $W$ as the large rectangle of Figure 2. In the construction of the $(n+1)$ th stage, we subdivide the interval $\left[0,1 / r_{2 n}\right]$ into $q_{2 n+1}$ intervals and take the leftmost subinterval of length $1 / r_{2 n+2}$ of each such interval. Similarly we subdivide $\left[0,1 / r_{2 n+1}\right]$. Thus we obtain $q_{2 n+1}$ stacks of $q_{2 n+2}$ rectangles. A typical rectangle in the $j$ th stack $\left(j=0,1,2, \ldots, q_{2 n+1}-1\right)$ is of the form

$$
\begin{aligned}
B_{j, k}=\left[j / r_{2 n+1}, j / r_{2 n+1}+1 / r_{2 n+2}\right] \times\left[k / r_{2 n+2}, k / r_{2 n+2}+1 / r_{2 n+3}\right] & \\
k & =0,1,2, \ldots, q_{2 n+2}-1 .
\end{aligned}
$$

Then

$$
P\left(B_{j, k}\right)=\left[j / r_{2 n+1}+k / r_{2 n+2}, j / r_{2 n+1}+(k+1) / r_{2 n+2}\right] \times\left[0,1 / r_{2 n+3}\right],
$$

and we see that the $P\left(B_{j, k}\right)$ are nonoverlapping. Furthermore, we find

$$
\bigcup\left\{P\left(B_{j, k}\right): k=0,1, \ldots, q_{2 n+2}-1\right\}=\left[j / r_{2 n+1},(j+1) / r_{2 n+1}\right] \times\left[0,1 / r_{2 n+3}\right],
$$

and it then follows that

$$
\bigcup\left\{P\left(B_{j, k}\right): k=0,1, \ldots, q_{2 n+2}-1, j=0,1, \ldots, q_{2 n+1}-1\right\}=W .
$$

This then establishes the proposition.

From the above, as in (3.4), we can now show that $\mu_{1, n} * \mu_{2, n}=\lambda$ for

$$
\text { (3.9) } \begin{aligned}
\left(\mu_{1, n} * \mu_{2, n}\right)(E) & =\left(\mu_{1, n} \times \mu_{2, n}\right)\left(p^{-1}(E)\right)=r_{2 n+1}(\lambda \times \lambda)\left(p^{-1}(E) \cap Q_{n}\right) \\
& =r_{2 n+1} \cdot \lambda(E) \cdot\left(1 / r_{2 n+1}\right)=\lambda(E) \quad(\bmod 1) . \quad \square
\end{aligned}
$$

Suppose now that $m \geq n$. Then the support of $\mu_{1, n} \times \mu_{2, m}$ lies in the support of $\mu_{1, n} \times \mu_{2, n}$ and is obtained by successively subdividing the intervals $I_{1, n}$ on the vertical axis. Thus we subdivide each rectangle into horizontal strips. The projection of these strips will be several horizontal strips $S_{n, m}$ in $Q_{n}$ (since each rectangle projects into $Q_{n}$ ). The mass of each strip is the same and their total mass is one (the $\mu_{i, n}$ are probability measures). One then easily sees that

$$
\begin{aligned}
\mu_{1, n} * \mu_{2, m}(E) & =\left(\mu_{1, n} \times \mu_{2, m}\right)\left(p^{-1}(E) \cap\left(\bigcup S_{n, m}\right)\right) \\
& =\left(\mu_{1, n} \times \mu_{2, n}\right)\left(p^{-1}(E) \cap Q_{n}\right)=\lambda(E) \text { by }(3.9) .
\end{aligned}
$$

Finally, for the case $m \leq n$, we can project on to the vertical axis and use a similar argument. This completes the proof of Theorem 3.1.

Combining Corollary 2.6, Proposition 2.7 and Theorem 3.1, we obtain the following

3.11 THEOREM. There are compact subsets $E_{1}$ and $E_{2}$ of the unit interval, each of capacity zero, such that the convolution of the natural Evans measures $\mu_{1}$ and $\mu_{2}$ supported on the sets $E_{1}$ and $E_{2}$ is Lebesgue measure.

It is worth remarking that the measures $\mu_{1}$ and $\mu_{2}$ both have infinite energy, while their convolution is Lebesgue measure on $I$ and so has finite energy. Also it is not difficult to see that this result is true in all dimensions. For instance,

$$
\left(E_{1} \times E_{1} \times \cdots \times E_{1}\right)+\left(E_{2} \times E_{2} \times \cdots \times E_{2}\right)=I^{n}
$$

and for a given $\Phi$ the Hausdorff $\Phi$-measure of $E_{i} \times E_{i} \times \cdots \times E_{i}$ can be made arbitrarily small by assuring that $E_{i}$ is sufficiently small, $i=1,2$. That the natural 
probability measures supported on $E_{i} \times E_{i} \times \cdots \times E_{i}, i=1,2$, convolve to give the usual Lebesgue measure of the cube $I^{n}$ is not difficult and we leave the details to the reader.

An alternative proof of Theorem 3.1, using probability theory, has been pointed out to us by $\mathrm{R}$. Lyons. We omit the technical details and briefly outline the method here. One can show that

$$
\lambda=\underset{i=1}{\stackrel{\infty}{*}} S_{i}, \quad \text { where } S_{i}=\sum_{p=0}^{q_{i}-1}\left(\frac{1}{q_{i}}\right) \delta\left(\frac{p}{r_{i}}\right) .
$$

To see this, we consider the successive $p_{i}$ in the expansion of a point $x$ (see (1.1)) to be a type of decimal expansion. We then attach equal mass at each possible point of such an expansion. A probabilistic argument then shows that this is indeed Lebesgue measure. Similarly, we find

$$
\begin{aligned}
& \mu_{1, n}=\left(\underset{1 \leq k \leq 2 n+1}{\stackrel{k \text { odd }}{*}} S_{k}\right) *\left(\begin{array}{c}
\underset{*}{*} \\
j>2 n+1
\end{array} S_{j}\right), \\
& \mu_{2, n}=\left(\underset{2 \leq k \leq 2 n+2}{\stackrel{\substack{\text { even } \\
*}}{*}} S_{k}\right) *\left(\underset{j>2 n+2}{\stackrel{\infty}{*}} S_{j}\right) .
\end{aligned}
$$

This is seen in much the same way as (3.12). Now it is true that all the infinite convolutions here converge unconditionally. Hence in the convolution $\mu_{1, n} * \mu_{2, m}$ we may rearrange terms to obtain Lebesgue measure (as in (3.12)) convolved with an error term from the extra factors, see, for instance, Chapter 6 of [GM]. However, convolving any measure with Lebesgue measure (on the circle) yields Lebesgue measure and so we find that $\mu_{1, n} * \mu_{2, m}=\lambda(\bmod 1)$ as above.

4. In the last section we found a nontrivial factorization of Lebesgue measure. This is, neither of the measures $\mu_{1}$ nor $\mu_{2}$ are point masses. We now consider what other measures could possibly be factorized in such a fashion and how Lebesgue measure can be determined from the measures $\mu_{1}$ and $\mu_{2}$. Throughout this section we fix two sets $E_{1}$ and $E_{2}$ as constructed in $\S 1$.

Given a nonnegative Lebesgue integrable function $f$ defined on the unit interval with $\int f d \lambda=1$, we obtain a probability measure $f(x) d \lambda(x)$. Suppose we could factor $f d \lambda$ as

$$
f(x) d \lambda(x)=\eta_{1}(x) * \eta_{2}(x),
$$

where $\eta_{1}$ and $\eta_{2}$ are probability measures supported on the sets $E_{1}$ and $E_{2}$, respectively.

4.2 PROPOSITION. With the above assumptions, there is a $\mu_{1}$-measurable function $g$ such that

$$
d \eta_{1}(x)=g(x) d \mu_{1}(x)
$$

and a $\mu_{2}$-measurable function $h$ such that

$$
d \eta_{2}(x)=h(x) d \mu_{2}(x) .
$$


ProOF. Equation (4.1) implies that for any Lebesgue measurable set $F$ contained in the interval $I$,

$$
\lambda_{f}(F)=\int_{F} f(t) d \lambda(t)=\iint_{E_{1} \times E_{2}} \chi_{F}(x+y) d \eta_{1}(x) d \eta_{2}(y) .
$$

We recall that $E_{1}+E_{2}=I$. Given two Borel subsets $F_{1}$ and $F_{2}$ of $E_{1}$ and $E_{2}$ we set $F=F_{1}+F_{2}$. It easily follows that

$$
\chi_{F}(x+y)=\chi_{F_{1}}(x) \cdot \chi_{F_{2}}(y)=\chi_{\left(F_{1} \times F_{2}\right)}(x, y) \text { for } x \in E_{1} \text { and } y \in E_{2} .
$$

The above, together with (4.3) and Fubini's Theorem, yield

$$
\lambda_{f}(F)=\eta_{1}\left(F_{1}\right) \cdot \eta_{2}\left(F_{2}\right),
$$

and consequently

$$
\eta_{1}\left(F_{1}\right)=\lambda_{f}\left(F_{1}+E_{2}\right) \text { and } \eta_{2}\left(F_{2}\right)=\lambda_{f}\left(F_{2}+E_{1}\right) .
$$

We now define

$$
g(x)=\int_{E_{2}} f(x+y) d \mu_{2}(y) \text { for } x \in E_{1}
$$

and

$$
h(y)=\int_{E_{1}} f(x+y) d \mu_{1}(x) \text { for } y \in E_{2} .
$$

By Fubini's Theorem, $g$ is $\mu_{1}$-measurable and $h$ is $\mu_{2}$-measurable. Furthermore, we find from the above (recalling $\mu_{1} * \mu_{2}=\lambda$ )

$$
\begin{aligned}
\eta_{1}\left(F_{1}\right) & =\lambda_{f}\left(F_{1}+E_{2}\right)=\iint_{E_{1} \times E_{2}} \chi_{F_{1}}(x) f(x+y) d \mu_{1}(x) d \mu_{2}(y) \\
& =\int_{F_{1}} g(x) d \mu_{1}(x) .
\end{aligned}
$$

Similarly we find

$$
\eta_{2}\left(F_{2}\right)=\int_{F_{2}} h(y) d \mu_{2}(y)
$$

This completes the proof.

Next Proposition 4.2 and (4.3) yield

$$
\begin{aligned}
\iint_{E_{1} \times E_{2}} \chi_{F}(x+y) f(x+y) d \mu_{1}(x) d \mu_{2}(y) \\
\quad=\iint_{E_{1} \times E_{2}} \chi_{F}(x+y) g(x) h(y) d \mu_{1}(x) d \mu_{2}(y)
\end{aligned}
$$

for all Borel subsets $F$ of $I$.

It is not difficult to see that (4.4) implies that continuous functions of the form $f(x+y)$ are dense in the space of continuous functions on $E_{1} \times E_{2}$. Consequently from (4.8) we find

$$
f(x+y)=g(x) h(y), \quad(x, y) \in E_{1} \times E_{2} \text { a.e. w.r.t. } \mu_{1} \times \mu_{2} .
$$

At a point $(a, b)$ where $(4.9)$ is satisfied we find

$$
f(y+a)=g(a) h(y) \text { a.e. } \mu_{2} \text { and } f(x+b)=h(b) g(x) \text { a.e. } \mu_{1} .
$$


Thus $g$ and $h$ are determined by the values of $f$ on the sets $E_{1}$ and $E_{2}$. Let us assume for the sake of simplicity that $(a, b)=(0,0)$ and that $f(0)=g(0)=h(0)=1$. Then (4.9) becomes

$$
f(x+y)=f(x) f(y) \text { a.e. } \mu_{1} \times \mu_{2} .
$$

If $f$ is a differentiable function of the interval, then (4.11) easily yields $f(t)=c e^{d t}$. Equation (4.11) is indeed very restrictive, in general, and leads one to suspect that there are very few measures of the form $f d \lambda$ which can be factored via the convolution of two such measures.

One can actually find solutions to (4.11) which are continuous except at a countable number of points as follows:

Let $g$ and $h$ be continuous functions on $E_{1}$ and $E_{2}$, respectively (where the topology of $E_{1}$ and $E_{2}$ is the topology they inherit as subspaces of $\left.[0,1]\right)$. We would like to define a continuous function $f$ on the interval $I$, by the rule

$$
f(t)=g(x) \cdot h(y)
$$

where $t=x+y, x \in E_{1}$ and $y \in E_{2}$.

Thinking of the expansion of a point $t \in I$ (see (1.1)) as a "decimal" expansion $t=\left(0 . p_{1} p_{2} p_{3} \ldots\right)$, we see by definition the sets $E_{1}$ and $E_{2}$ are the points with expansion

$$
x \in E_{1} \Rightarrow x=\left(0 . p_{1} 0 p_{3} 0 p_{5} 0 \ldots\right), \quad \text { "zeros in the even slots", }
$$

and

$$
y \in E_{2} \Rightarrow y=\left(0.0 p_{2} 0 p_{4} 0 \ldots\right), \quad \text { "zeros in the odd slots". }
$$

This gives us two functions $t \rightarrow x(t)$ and $t \rightarrow y(t)$ defined by the above splitting of the expansion. However, the expansion of a point $t \in I$ need not be unique (as in the case of repeating 9's in a decimal expansion) so that a priori the functions $x(t)$ and $y(t)$ are not well defined. We can alleviate this problem by identifying the points

$$
\left(0 . p_{1} p_{2} \ldots p_{n}\left(q_{n+1}-1\right)\left(q_{n+2}-1\right) \ldots\right) \text { and }\left(0 . p_{1} p_{2} \ldots\left(p_{n}+1\right) 000 \ldots\right),
$$

where $p_{n}$ is the last integer in the expansion with $p_{i}<q_{i}-1$, and always agree to choose the latter expansion for such a point $t$. This then makes the expansion unique and the functions $x(t)$ and $y(t)$ well defined on $I$. Using the rule suggested in (4.12) we can define a function on the interval as

$$
f(t)=g(x(t)) \cdot h(y(t)) .
$$

The function $f$ will be continuous except at a countable number of points provided we show that the functions $x(t)$ and $y(t)$ are. We will show that $x(t)$ is continuous except at the points with expansion terminating in zeros. That $y(t)$ is continuous except at such points will follow in a similar fashion. Thus let $t$ be a point of $I$ whose expansion does not terminate in zeros and let $\delta>0$. Let $N$ be so large that $1 / r_{N}<\delta$. Next, by our choice of the expansion of $t$, there is an $i>N$ such that $0<p_{i}<q_{i}-1$. We accordingly set $\tau=1 / r_{i}$. We now see that if $\left|t-t^{\prime}\right|<\tau$, then the expansions of $t$ and $t^{\prime}$ agree to the first $i-1$ places and hence so do the expansions of $x(t)$ and $x\left(t^{\prime}\right)$. Thus

$$
\left|x(t)-x\left(t^{\prime}\right)\right|<1 / r_{i-1} \leq 1 / r_{N}<\delta .
$$


It is not difficult to see that the functions $x(t)$ and $y(t)$ are not continous at points whose expansion terminates in zeros since a small perturbation will carry far up the expansion, and so change $x(t)$ quite a bit.

The following result follows easily from the above.

4.13 THEOREM. If $f$ is a nonnegative continuous function on $I$, then

$$
f d \lambda=\eta_{1} * \eta_{2}
$$

where $\eta_{1}$ and $\eta_{2}$ are measures supported on $E_{1}$ and $E_{2}$, respectively, only if there are continuous functions $g$ and $h$ defined on $E_{1}$ and $E_{2}$, respectively, such that $f(t)=g(x(t)) \cdot h(y(t))$.

It seems quite plausible that the only such functions $f$ are exponentials; unfortunately, we were unable to decide this.

ADDED IN PROOF. A Tortrat has recently proven the conjecture stated after Theorem 4.13. The authors would also like to thank Professor Tortrat for his valuable comments.

\section{REFERENCES}

[E] H. G. Eggleston, Note on certain s-dimensional sets, Fund. Math. 36 (1949), 40-43.

[GM] C. G. Graham and O. C. McGehee, Essays in commutative harmonic analysis, Grundlehren Math. Wiss., 238, Springer-Verlag, Berlin, Heidelberg and New York, 1979.

[KS] J-P. Kahane and R. Salem, Ensembles parfaits et séries trigonométriques, Actualités Sci. Indust., no. 1301, Hermann, 1963.

[N] R. Nevanlinna, Analytic functions, Grundlehren Math. Wiss., 162, Springer-Verlag, Berlin, Heidelberg and New York, 1970.

[T] A. Tortrat, Classes de mesures singulières sur la droite, convolutions infinies de Wintner et lois indéfiniment divisibles, J. Math. Pures Appl. 39 (1960), 231-273.

Department of Mathematics, Wellesley College, Wellesley, MassachuSETTS 02181 (Current address of N. Levenberg, Jr.)

Department of mathematics, Yale University, New haven, Connecticut 06520 (Current address of G. J. Martin)

Department of Mathematics, University of Michigan, ANn ARbor, Michigan 48109 (Current address of A. L. Shields and S. Zdravkovska) 